\title{
DESIGN OF THEORETICALLY OPTIMAL THERMOACOUSTIC COOLING DEVICE
}

\author{
Tomáš Tisovskýa, ${ }^{\mathrm{a}, 1}$, Tomáš Vit ${ }^{\mathrm{b}, 1}$ \\ ${ }^{1}$ Department of Power Engineering Equipment, Faculty of Mechanical Engineering, Technical University of Liberec, Czech Republic
}

\begin{abstract}
The aim of this article is to design theoretically optimal thermoacoustic cooling device. The opening chapter gives the reader brief introduction to thermoacoustic, specializing in the thermoacoustic principle in refrigerator regime. Subsequent part of the article aims to explain the principle on which thermoacoustic is simulated in DeltaEC. Numbers of executed numerical simulations are listed and the resulting thermoacoustic cooling device design is presented along with its main operation characteristics. In conclusion, recommendations for future experimental work are given and the results are discussed.
\end{abstract}

\section{Thermoacoustic as an alternative cooling technology}

Thermoacoustics was defined by Rott [1] as a subject dealing generally with effects in acoustics in which heat conduction and entropy variations of a medium are of importance.

Acoustic wave spreading throughout a fluid is a phenomenon composed out of variations of pressure and velocity. These pressure variations are usually small enough for the wave to be considered an isoentropic process. If the fluid under the influence of acoustic wave is left in thermal contact with constant temperature solid boundary, heat transfer between solid and fluid will take place resulting in the thermoacoustic effect.

It was found out that device can be made in which the termoacoustic effect can be used to transfer heat from low potential source into high potential heat sink. Energy used to pump the heat is supplied by the acoustic wave induced in the fluid by external source such as loudspeaker.

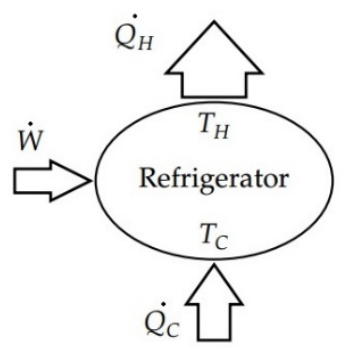

Figure 1. Flows of work and heat inside a thermoacoustic refrigerator [2]

\footnotetext{
${ }^{\mathrm{a}}$ Corresponding author: tomas.tisovsky@tul.cz

b Corresponding author: tomas.vit@tul.cz
}

\section{Introduction to thermoacoustics}

To explain the thermoacoustic effect in more detail it is convenient to imagine a thin stationary solid plate surrounded by volume of fluid subjected to oscillatory boundary condition in a way that the resulting acoustic wave propagates in the direction of the longest plate dimension.

Such simple device will perform as a refrigerator (i.e. transferring heat in an initially uniform temperature plate from one end to the other) only as long as the temperature gradient alongside the plate is small enough. The process which single parcel of working fluid undergoes is shown in Figure $2 \mathrm{a}$.

The process which every parcel of fluid undergoes within a piston movement period consists of two reversible adiabatic steps 1-2 and 3-4 and two isobaric heat transfer steps 2-3 and 4-1.

At the beginning of the cycle, the piston moves to the right and compresses the parcel which consequently warms up. In thermoacoustic refrigeration cycle, the parcel is now warmer that the adjacent part of the solid plate and heat flows irreversibly from parcel to plate. Next the piston moves to the left to its original position and the parcel expands and cools in process. Again, if the temperature gradient along the plate is small enough, heat flows irreversibly, this time from the solid plate into the parcel. After that the parcel is in its initial position and the cycle starts again.

The amount of heat which is taken from/given to the plate at during one cycle is quite small and many cycles are needed for a substantial temperature gradient to build up along the plate.

It is also notable that the acoustic work consumed to compress the gas in step 1-2 is of the same amount as 
work which is returned by expanding gas in step 3-4. Therefore no net work is consumed in those steps. Net work is consumed only in steps 2-3 and 4-1 in which piston has to move a little bit to keep the pressure throughout the gas constant.

(a) Refrigerator

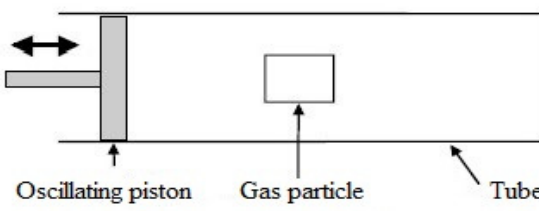

1

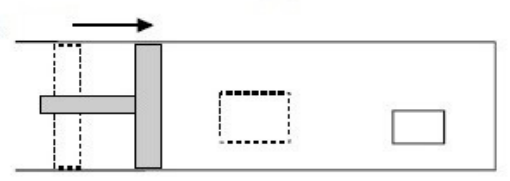

2

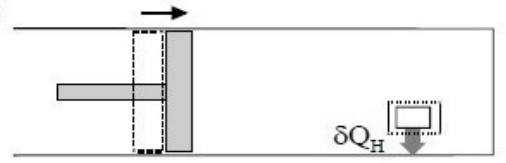

3
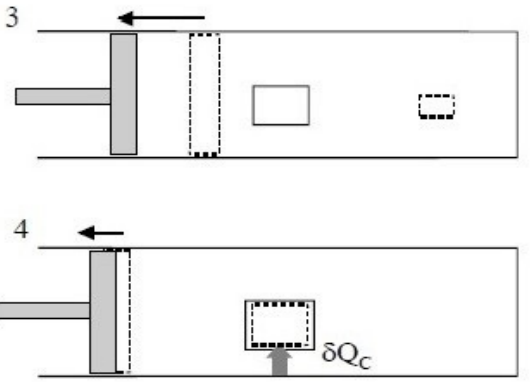

(b)

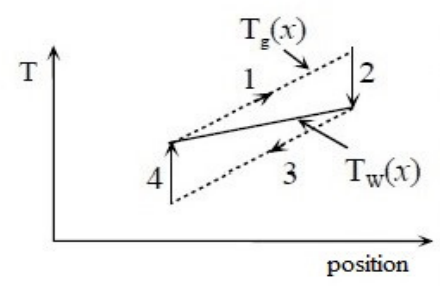

Figure 2. a) a typical gas parcel executing the four step cycle in a thermoacoustic refrigerator

b) gas and wall temperature plotted versus the position in resonator, [2]

The time lag between displacement and heat transfer is necessary for the operation of thermoacoustic device and is caused by the imperfect thermal contact between solid and gas. Sound wave at greater distances from solid boundary is, as is mentioned above, adiabatic process and if the gas and plate were at a zero distance from each other, the parcel would simply be compressed and expanded isothermally. Parcel that are the most effective to the thermoacoustic effect are located approximately in a distance about the thermal penetration depth $\delta_{\mathrm{k}}$ away from the solid boundary.

The thermal penetration depth is defined as

$$
\delta_{\mathrm{k}}=\sqrt{\frac{\mathrm{k}_{\mathrm{g}}}{\pi \mathrm{f} \rho_{\mathrm{m}} \mathrm{c}_{\mathrm{p}}}},
$$

where $\mathrm{k}_{\mathrm{g}}$ is the thermal conductivity of a working gas, $\rho_{\mathrm{m}}$ is its mean density and $c_{p}$ is its specific heat capacity. It is clear from previous discussion that it is desirable to have as much of a working gas volume as possible in appropriate, relatively small, distance away from solid boundary on which temperature gradient is imposed. Way to achieve this is to place a component called stack downstream of the source from which the acoustic wave is propagating. Stack consists of many parallel channels aligned in a direction of the acoustic wave motion giving the passing wave large surface to interact with.

The main parts combining the thermoacoustic cooling device are therefore: resonator, loudspeaker, stack, hot heat exchanger and cold heat exchanger. The loudspeaker at one end of the resonator gives rise to the acoustic wave which then propagates through resonator to its other end where it reflects. Characteristics of the reflected wave are given by the characteristics of the incident wave as well as by the type of boundary at the point of reflection (closed end / open end).The incident wave and the reflected wave interfere in the resonator resulting in the standing wave pattern. Generally, the incident and the reflected wave do not form the standing wave pattern. This has to be achieved by correctly choosing oscillation frequency for a given apparatus.

In standing wave based thermoacoustic devices, the heat flow due to thermoacoustic effect is always in the direction towards the nearest pressure antinode.

The remaining parts of the thermoacoustic cooling device, which have not been discussed, are the heat exchangers. The hot heat exchanger is located closer to the loudspeaker and its purpose is to maintain proper temperature on the hot end of a stack, usually the ambient temperature of about $300 \mathrm{~K}$ should suffice. The cold heat exchanger, on the other hand, is design to heat up the working gas enough to maintain the desired cooling temperature.

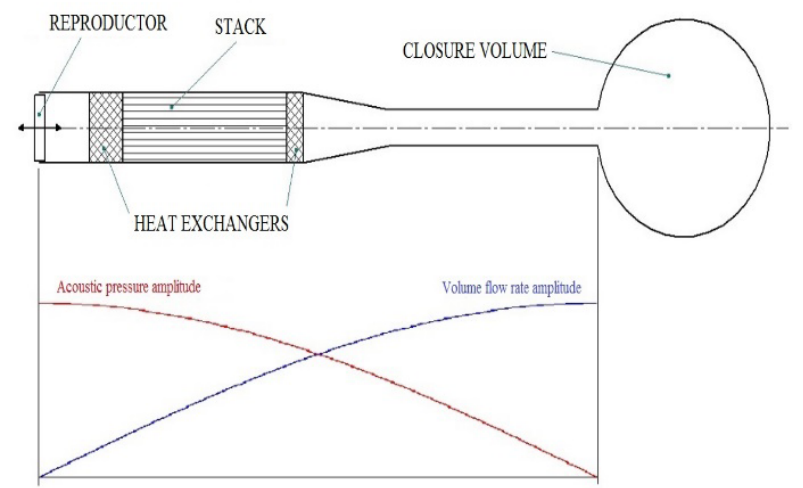

Figure 3. Resonator scheme 


\section{Design of thermoacoustic cooling device in DeltaEC}

DeltaEC - Design Environment for Low-Amplitude Thermoacoustic Energy Conversion, is a computer program that can calculate details of how thermoacoustic equipment performs, or can help the user to design equipment to achieve desired performance. [4]

DeltaEC integrates in one spatial dimension using lowamplitude acoustic approximation and sinusoidal time dependence. It integrates up to four, two of which are complex, equations in gas in a geometry given by the user as a sequence of segments. The four equations can are integrated to obtain complex acoustic pressure amplitude, complex volume flow rate amplitude, mean temperature and total power flow.

Apart from few smaller exceptions DeltaEC does not include any nonlinear effects which arise at high pressure amplitudes. As a consequence care must be taken to choose the maximum pressure amplitude, which occurs at the loudspeaker, such that two conditions hold.

a) Condition (2) for the acoustic Mach number, given by means of maximum acoustic pressure amplitude $\mathrm{p}_{01}$ and the square of velocity of sound in gas a, is

$\mathrm{M}_{\mathrm{T}}=\frac{\mathrm{p}_{01}}{\rho_{\mathrm{m}} \mathrm{a}^{2}}=0,1$.

b) The Reynolds number based on Stokes boundary layer thickness $\delta_{v}$ is less than a value given by equation (3), which is the condition for transition to turbulence in boundary layer

$\operatorname{Re}^{\delta_{v}}=\frac{\mathrm{u}_{1} \delta_{v}}{v}=550$.

Figure 4 shows a simplified model of thermoacoustic cooling device. In reality, model also includes many RPN (Reversed Polish Notation) math segments which are used for better control of the model behaviour. Main parts of the model are listed in top left corner of the figure 4 . The begin segment is used to define 6 real boundary conditions needed for an unique solution of the equations (2) to (5) to exist. Model below was designed so that few boundary conditions were given elsewhere from the begin segment hence a shooting method needs to take place. Shooting method algorithm is adjusting variables called guesses which are selected by the user to gain a correct value of variable called targets (i.e. the boundary conditions).

The guesses are inlet volume flow rate amplitude, phase shift and magnitude of the loudspeaker source voltage, heat flow from/to both heat exchangers and length of two resonator ducts.

The targets are resonance condition, temperatures of the working gas at exchangers' levels, value of the maximum acoustic pressure amplitude, position of the center of the stack and the conditions that ensure closure and isolation of the resonator at the compliance end.

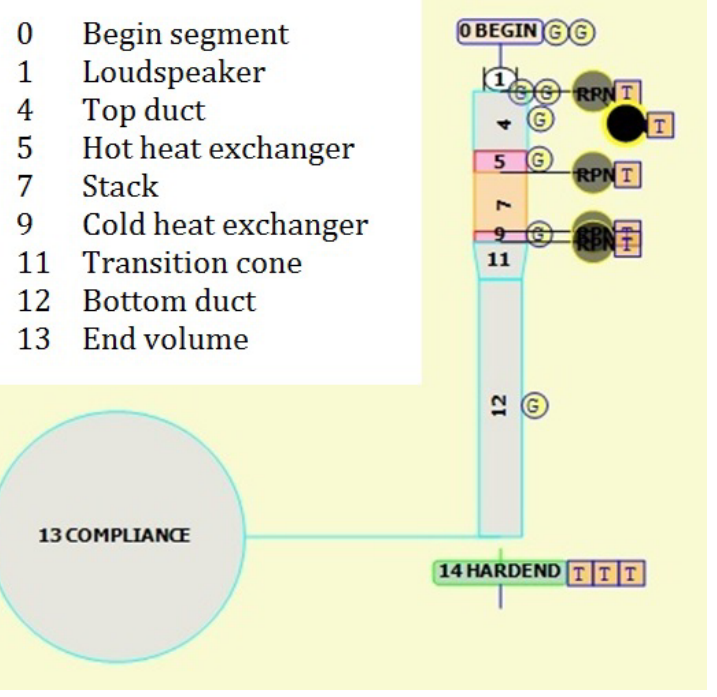

Figure 4. Composition of numerical model in DeltaEC

Series of simulations was carried out, namely the analysis of the appropriate end volume diameter, analysis of the stack plate half-spacing magnitude, analysis of the optimal stack center position in the resonator, comparison of different stack materials, analysis of the influence of various resonator duct diameter, optimal stack length analysis and analysis of the appropriate narrowing of the resonator duct. Most of these analyses were evaluated with respect to the highest possible coefficient of performance.

Table 1. Parameters of the resulting device

\begin{tabular}{|c|c|c|c|c|c|c|}
\hline \multicolumn{2}{|c|}{$\begin{array}{l}\mathrm{p}_{\mathrm{m}} \\
{[\mathrm{MPa}]}\end{array}$} & $\begin{array}{l}\mathrm{f} \\
{[\mathrm{Hz}]}\end{array}$ & \multicolumn{2}{|c|}{$\begin{array}{l}\mathrm{p}_{01} \\
{[\mathrm{kPa}]}\end{array}$} & $\begin{array}{l}\mathrm{L}_{\text {resonator }} \\
{[\mathrm{mm}]}\end{array}$ & $\begin{array}{l}\frac{x_{\text {gtack center }}}{L_{\text {resonator }}} \\
{[1]}\end{array}$ \\
\hline 1,2 & & 500 & 12 & & 416 & 0,25 \\
\hline $\begin{array}{l}\mathrm{D}_{\text {reso }} \\
{[\mathrm{mm}]}\end{array}$ & ator & $\begin{array}{l}\frac{d_{\text {duct }}}{D_{\text {duct }}} \\
{[1]}\end{array}$ & & $\begin{array}{l}\text { mpliance } \\
\text { n] }\end{array}$ & $\begin{array}{l}\frac{y_{0}}{\delta_{k}} \\
{[1]}\end{array}$ & $\begin{array}{l}\mathrm{y}_{0} \\
{[\mathrm{~mm}]}\end{array}$ \\
\hline 34 & & 0,78 & 10 & & 2,64 & 0,23 \\
\hline $\begin{array}{l}\mathrm{T}_{\mathrm{C}} \\
{[\mathrm{K}]}\end{array}$ & $\begin{array}{l}\text { COP } \\
{[1]}\end{array}$ & $\begin{array}{l}\frac{\mathrm{C}}{\mathrm{CO}} \\
{[1]}\end{array}$ & & $\begin{array}{l}\eta_{\text {ea }} \\
{[1]}\end{array}$ & $\begin{array}{l}\dot{W}_{\mathrm{el}} \\
{[\mathrm{W}]}\end{array}$ & $\begin{array}{l}\dot{\mathrm{E}} \\
{[\mathrm{W}]}\end{array}$ \\
\hline 260 & 0,79 & 0,1 & & 0,54 & 428 & 229 \\
\hline $\begin{array}{l}\mathrm{Q}_{\mathrm{C}} \\
{[\mathrm{W}]}\end{array}$ & Q & & $\begin{array}{l}\mathrm{Re}^{\delta_{\mathrm{v}}} \\
1]\end{array}$ & $x_{-}$stack & $\begin{array}{l}\operatorname{Re}^{\delta_{v_{\text {max }} r}} \\
{[1]}\end{array}$ & \\
\hline 182 & 61 & & 235 & & 594 & \\
\hline
\end{tabular}

Table 1 shows the resulting resonator dimensions and its working parameters. Variable $\eta_{\text {ea }}$ stands for the efficiency of transformation of electric energy of loudspeaker source to acoustic energy and it is highest 
possible for given device. Variable $\mathrm{y}_{0}$ is the stack plate half-spacing.

One of the greatest advantages of thermoacoustic refrigerator is the ease in which it can be adjusted to work optimally in various cooling temperature modes. Cooling power corresponding with given temperature can be than easily adjusted by imposing different acoustic pressure amplitude. Tables 2 and 3 show the difference between simple adjustment of a stack position when cooling to different temperature and leaving the original resonator unchanged.

Table 2. Resonator parameters corresponding to various cooling temperature - original resonator

\begin{tabular}{|c|c|c|c|}
\hline & \multicolumn{3}{|c|}{ original resonator } \\
\hline $\begin{array}{l}\mathbf{T}_{\mathrm{C}} \\
{[\mathrm{K}]}\end{array}$ & \begin{tabular}{|l}
$\mathrm{f}$ \\
{$[\mathrm{Hz}]$}
\end{tabular} & $\begin{array}{l}\varepsilon_{\mathrm{ch}} \\
{[1]}\end{array}$ & $\begin{array}{l}\dot{Q}_{C} \\
{[W]}\end{array}$ \\
\hline 290 & 526 & 1,26 & 358 \\
\hline 270 & 509 & 0,97 & 239 \\
\hline 250 & 491 & 0,59 & 126 \\
\hline 230 & 472 & 0,10 & \begin{tabular}{|l|}
17 \\
\end{tabular} \\
\hline
\end{tabular}

Table 3. Resonator parameters corresponding to various cooling temperature - adjusted resonator

\begin{tabular}{|l|l|l|l|l|l|l|}
\hline \multicolumn{7}{|c|}{ resonator with optimal stack position } \\
\hline $\begin{array}{l}\mathrm{T}_{\mathrm{C}} \\
{[\mathrm{K}]}\end{array}$ & $\begin{array}{l}\frac{\mathbf{x}_{\mathbf{s}}}{\mathrm{L}} \\
{[\%]}\end{array}$ & $\begin{array}{l}\mathrm{L}_{\mathrm{rez}} \\
{[\mathrm{mm}]}\end{array}$ & $\begin{array}{l}\mathbf{x}_{\mathrm{s}} \\
{[\mathrm{mm}]}\end{array}$ & $\begin{array}{l}\mathrm{f} \\
{[\mathrm{Hz}]}\end{array}$ & $\begin{array}{l}\boldsymbol{\varepsilon}_{\mathrm{ch}} \\
{[1]}\end{array}$ & $\begin{array}{l}\mathbf{Q}_{\mathrm{C}} \\
{[\mathrm{W}]}\end{array}$ \\
\hline 290 & 38 & 434 & 166 & 500 & 1,60 & 495 \\
\hline 270 & 28 & 422 & 120 & 500 & 1,01 & 251 \\
\hline 250 & 22 & 411 & 90 & 500 & 0,62 & 133 \\
\hline 230 & 18 & 379 & 71 & 500 & 0,36 & 70 \\
\hline
\end{tabular}

\section{Conclusions}

Designed thermoacoustic cooling device has several advantages compared to conventional cooling devices, mainly it is the ease of proportional control, very simple structure and the fact that they are environmentally friendly. Moreover such device would occupy a relatively small space and surprisingly enough, it would be silent. This is due to the fact that the working gas inside the thermoacoustic cooling device is pressurized and therefore the ratio of the acoustic pressure to the mean pressure would be small even for amplitudes of acoustic pressure that would be considered high in atmospheric conditions. The only significant source of noise can therefore only be the mechanical vibrations of the whole device and such problem can be easily solved by more suitable connection of the device to its base.
For their advantages, thermoacoustic cooling devices can be suitable to use in relatively extreme conditions such are in space or in Third world countries.

On the other hand, there are also some disadvantages of thermoacoustic cooling technology. The main disadvantage is the poor efficiency exhibited by thermoacoustic cooling devices. This might be resolved once a better understanding of involved physics is obtained, mainly the understanding of non-linear effects accompanying coupled heat transfer and acoustics. There are also technological and construction aspects of improving efficiency of thermoacoustic devices since present heat exchangers and stack are still hardly optimal. The device proposed in this article could be used, for example, in food merchandising, where toxicity of a conventionally used refrigerant can be a problem.

At the end, it is worth mentioning that thermoacoustic cooling devices can be constructed so that they can work without a loudspeaker or similar device (acoustic wave can be generated only by stack along which is maintained a proper temperature gradient), or they can be constructed so that they can directly cool the gas which requires cooling (no additional heat exchanger is required).

\section{References}

[1] Rott, N. (1980). "Thermoacoustics". Adv. Appl. Mech. 20 (135): 135. doi: $10.1016 / \mathrm{S} 0065-2156(08) 70233-3$

[2] In't Panhuis P. H. M. W. Mathematical Aspects of Thermoacoustics [online]. Universiteit Eindhoven: Print Service Technische, 2003, 190 s. ISBN 97890-386-1862-3.

[3] Tijani, Moulay El Hassan. Loudspeaker-driven thermo-acoustic refrigeration. Eindhoven: Technische Universiteit Eindhoven, 2001, $170 \mathrm{~s}$. ISBN 90-386-1829-8. 